\title{
FAM98A associates with DDX1-C14orf166-FAM98B in a novel complex involved in colorectal cancer progression
}

Khondker Ayesha Akter1, Mohammed A. Mansour2, Toshinori Hyodos, Takeshi Senga3

1Department of Pharmaceutical Sciences, North South University Bangladesh, Dhaka-1229, Bangladesh

${ }_{2}$ Biochemistry Division, Department of Chemistry, Faculty of Science, Tanta University, Tanta 31527, Egypt

${ }_{3}$ Division of Cancer Biology, Nagoya University Graduate School of Medicine, 65 Tsurumai, Showa, Nagoya, 466-8550 Japan

Address correspondence to:

Mohammed A. Mansour; Biochemistry division, Department of Chemistry, Faculty of Science, Tanta University, Tanta 31527, Egypt; E-mail: biomansour@hotmail.com

Khondker Ayesha Akter; Department of Pharmaceutical Sciences, North South University Bangladesh, Dhaka-1229, Bangladesh; E-mail: ayash_8@yahoo.com

Running title: FAM98A \& B are required for PRMT1 expression

Keywords: Colorectal Cancer; FAM98A; FAM98B; PRMT1 


\begin{abstract}
Protein Arginine Methyl Transferase 1 (PRMT1) is deemed to be a potential oncogenic protein considering its overexpression in several malignancies including colorectal cancer. However, the molecular pathogenesis regarding PRMT1 overexpression and overall poor patient survival involved in this devastating and life threatening cancer remains obscured. In our previous study, we have identified FAM98A as a novel substrate of PRMT1 and also identified its role in ovarian cancer progression. Here, we showed that the two structural homologs FAM98A and FAM98B included in a novel complex with DDX1 and C14orf166 are required for PRMT1 expression. Analysis of the data from The Cancer Genome Atlas (TCGA) database and clinical colorectal cancer specimens also demonstrated a strong positive correlation and co-occurrence of PRMT1, FAM98A and FAM98B. These findings provide a mechanistic insight into how knockdown of FAM98A or FAM98B can suppress the malignant characteristics of cancer cells. Besides, we showed that FAM98A and FAM98B are working in the same axis as knockdown of both proteins together does not cause additional reduction in the cellular proliferation and colony formation of colorectal cancer cells.
\end{abstract}




\section{Introduction}

Colorectal cancer (CRC) is one of the most dominant types of cancer worldwide. It is the third most common cancer, the fourth most common reason of cancer death, and the second most common cancer with respect to the number of individuals surviving with cancer five years after diagnosis worldwide [1]. An estimated 1,361,000 people are diagnosed with CRC every year; approximately 694,000 people die from CRC annually; and 3,544,000 individuals are living with CRC [2]. Randomized controlled trials have shown that current and future levels of increased screening could provide for large reductions in CRC mortality. In several developed countries, organized, population-based screening programs have been imposed since 2006 [3]. Regardless of these efforts, new screening tools and therapeutic interventions are required to combat this deadly disease.

Protein arginine methylation is a common post translational modification in eukaryotes that has been implicated in several biological processes including signal transduction, gene transcription, DNA repair and mRNA splicing [4]. The major type of protein arginine (R) methyltransferase (PRMT), type I, transfers the methyl group from S-adenosyl-L-methionine (AdoMet) to the guanidino group of arginines in protein substrates, resulting in monomethylarginine and asymmetric dimethylarginine (ADMA) in substrate proteins [5]. Recent studies have linked this modification to carcinogenesis and metastasis. Alterations to the PRMTs sequences have not been found so far; however, over expression of these enzymes is very often associated with the progression of various cancers [6,7]. Among PRMT family proteins, PRMT1 is crucial for the majority (approximately 85\%) of methylated arginine in mammalian cells [4]. Evidences have demonstrated that PRMT1 is aberrantly expressed in various cancers, such as breast, prostate, lung, colon, ovarian and bladder cancer, as well as leukemia [8-16].

An increasing number of PRMT targets have been identified that are involved in a broad range of cellular processes, including DNA repair, transcriptional regulation, RNA processing and signal transduction [17]. Arginine residues methylated by PRMT1 as well as other PRMTs are mostly located in unique sequences that are glycine and arginine rich, called RGG/RG motifs or GAR motifs [18]. Although there are a number of proteins with RGG/RG repeats, whether arginine methylation of RGG/RG repeats by PRMT1 have important functions for the biological activities of these proteins or disease progression in human remains unknown. Many of PRMT1 substrates are key modulators of cancer cell growth, survival and invasion signaling [19]. Consistent with this concept, recently we have found that FAM98A which is a novel substrate of 
PRMT1 is required for cancer cell proliferation, migration and invasion [15]. However, the underline mechanism was elusive. Hence, we were interested to discover the novel protein complex including FAM98A and FAM98B essential for colorectal tumorigenesis. In this report, we have identified that FAM98A and FAM98B are significantly positively correlated with PRMT1 by analyzing the data from TCGA database and clinical CRC specimens. Upon depletion of either of these proteins, cancer cells' proliferation and anchorage-independent growth were significantly reduced. Also, a novel complex of FAM98A with DDX1, C14orf166 and FAM98B was found to be required for the proper expression of PRMT1 and in turn PRMT1mediated arginine methylation.

\section{Materials and Methods}

\subsection{Cells and antibodies}

Colorectal cancer cells, HCT116 and HT29 cells, were obtained from American Type Culture Collection (ATCC) and cultured in DMEM supplemented with 10\% fetal bovine serum (EQUITEC, Hendra, Australia). The cells were authenticated recently by short tandem repeat analysis using gene print巴 10 system (Promega, Madison, WI). The antibodies were purchased from the following manufacturers: anti-B-actin antibody, Sigma-Aldrich, St. Louis, MO, USA; anti-asymmetric dimethyl-arginine (ADMA) antibody, Active Motif, Carlsbad, CA, USA; AntiPRMT1 antibody, Cell Signaling, Danvers, MA, USA; Anti-FAM98A antibody, Aviva Systems Biology, San Diego, CA, USA; anti-GFP antibody, Neuro Mab (Davis, CA); anti-Flag antibody, Wako (Osaka, Japan); Anti-FAM98B antibody, Gene Tex, Irvine, California, USA.

\section{2. $c D N A$ constructs}

cDNAs for the human genes; PRMT1, FAM98A, FAM98B, DDX1 and C14orf166 were amplified by Polymerase Chain Reaction (PCR) from a cDNA library of HeLa cells. Each cDNA was cloned into the pQCXIP vector (TAKATA, Tokyo, Japan) with an N-terminal Flag or GFP tag. Also, several deletion mutants of FAM98A were generated by PCR.

\subsection{Western blot analysis}

Western blot analysis was performed as described previously [20]. In brief, cell lysates were loaded on SDS-polyacrylamide gels for electrophoresis (PAGE) and transferred to polyvinylidene difluoride (PVDF) membranes (Millipore, Billerica, MA, USA). The membranes were blocked with $1 \%$ skim milk for $1 \mathrm{~h}$ and then incubated with primary antibodies at $4^{\circ} \mathrm{C}$ overnight. Then, the membranes were washed with TBS-T buffer (10 mM Tris-HCl pH 7.4, 150 $\mathrm{mM} \mathrm{NaCl}, 0.05 \%$ Tween 20) for 15 min and incubated with HRP-labeled secondary antibodies. 
The signals were detected with the ECL system (Nacalai tesque, Kyoto, Japan). The signal intensities were estimated using Light Capture II equipped with CS analyzer (ATTO Corp., Tokyo, Japan).

\subsection{Patients and ethics statement}

Clinical colorectal cancer samples and normal colon samples were obtained from patients who went through surgery at the Nagoya University Hospital in 2012. For the detection of PRMT1, FAM98A and FAM98B expression by q-RT PCR, normal colon tissues and colorectal cancer tissues with stages II-IV were collected from male and female patients (Age 30-60 yr). The study was approved by the institutional review board of Nagoya University Graduate School of Medicine and conformed to the standards set by the Declaration of Helsinki. All participants of the study provided written informed consent to participate in this study.

\subsection{Quantitative PCR analysis}

Endogenous RNA was extracted using the RNeasy mini kit (Qiagen, Venlo, Netherlands) from colorectal cancer samples and cells, and then cDNAs were generated using primescript Reverse Transcriptase (TAKARA, Tokyo, Japan). The colorectal cancer specimens were obtained from patients who underwent surgery at the Nagoya University Hospital with informed consent. PCR was performed using the SYBR Premix Ex TaqTM II (TAKARTA), and the Thermal Cycler Dice TM Real Time System TP800 (TAKARA) was used for the analysis and quantification. The relative mRNA level was normalized to GAPDH. The sequences of primers used to amplify each gene were 5'-AGGTGGAGGAGTGGGTGTCGCTGTT-3’and 5'CCGGGAAACTGTGGCGTGATGG-3’ (GAPDH), 5'-CATCATGGAGGTGTCCTGTG-3’ and 5'GAAGAGGTGCCGGTTATGAA-3' (PRMT1)， 5'-AGGAAAACGTGCAAGCAACT-3' and 5'CACATCCCCAGATGTCAGTG-3' (FAM98A)， 5'-TTTGTCACCCAAGACAACGA-3' and 5'GCTGGTGCCACTACTTGTCC-3’ (FAM98B).

\section{6. siRNA transfection}

Knock down of the genes expression by siRNA transfection was performed as described previously [21]. The sequences of siRNA used to knockdown PRMT1, FAM98A and FAM98B were as follows: 5'-GAGGGCACAUCGUGACUGUGU-3' (PRMT1 siRNA), 5'CCAAACCUCCAGCCAAUAUTT-3' (FAM98A siRNA), and 5'GGAUGGUCUGAUAGAGCAATT-3' (FAM98B siRNA). The sequence of the control siRNA that targeted luciferase was 5'-CUUACGCUGAGUACUUCGATT-3'. All the siRNAs were obtained from Hokkaido System Sciences, Sapporo, Japan. The cells were transfected with $20 \mathrm{nM}$ of the 
siRNA using lipofectamine RNAiMAX (Invitrogen, Carlsbad, CA, USA) according to the manufacturer's instructions.

\subsection{Generation of stable cell lines}

HCT116 cells that constitutively expressed Flag-PRMT1 and Flag-FAM98A were generated by retrovirus infection. The pQCXIP vector encoding Flag-PRMT1or Flag-FAM98A was transfected into $293 \mathrm{~T}$ cells in combination with the pVPack-GP and pVPack-Ampho vectors (Stratagene, Tokyo, Japan) using Lipofectamine 3000 (Invitrogen, Carlsbad, CA). 48 hours after transfection, the supernatants were added to HCT116 cells with $2 \mu \mathrm{g} / \mathrm{mL}$ polybrene (Sigma-Aldrich, St. Louis, MO, USA), and infected cells were selected with $1 \mathrm{\mu g} / \mathrm{mL}$ puromycin for three days. The cells were cultured for $24 \mathrm{~h}$, and $1 \mathrm{\mu g} / \mathrm{mL}$ of puromycin (Sigma-Aldrich, St. Louis, MO, USA) was then added to the culture to select for the infected cells (gene expressing cells).

\subsection{Transfection and immunoprecipitation (IP)}

To detect the association of different proteins, 293T cells cultured in a $3.5-\mathrm{cm}$ dish were transfected with $1 \mathrm{\mu g}$ of each plasmid using Lipofectamine 3000 (Invitrogen, Carlsbad, CA, USA). After $24 \mathrm{~h}$, cells were washed twice with cold PBS and lysed in TNE buffer (50 mM Tris$\mathrm{HCl} \mathrm{pH} \mathrm{7.4,} 150 \mathrm{mM} \mathrm{NaCl}, 0.1 \% \mathrm{NP}$-40) with protease inhibitors for 15 minutes on ice and centrifuged at 15,000 rpm for 20 minutes to obtain clear cell lysates. The cell lysates were incubated with beads coupled with anti-Flag antibody at $4^{\circ} \mathrm{C}$ with rotation overnight. The Flagexpressing 293T cells were taken as control for IP analysis. The precipitates were washed with TNE buffer three times and suspended with Laemmli sample buffer for immunoblot analysis. Other experiments for interactions were performed with the same procedure and the experiments were performed twice to ensure the validity of the results.

\subsection{Protein identification by mass spectrometry (MS)}

Flag-FAM98A-expressing 293T cells were generated by retrovirus infection, while the Flagexpressing 293T cells were taken as control for mass spectrometry analysis. The pQCXIP vector encoding Flag or Flag-FAM98A was transfected into 293T cells in combination with the pVPack-GP and pVPack-Ampho vectors (Stratagene, Tokyo, Japan) using Lipofectamine 3000 (Invitrogen, Carlsbad, CA, USA). Forty-eight hours after transfection, the supernatants were added to $293 \mathrm{~T}$ cells with $2 \mathrm{\mu g} / \mathrm{mL}$ polybrene (Sigma-Aldrich), and infected cells were selected with $1 \mathrm{\mu g} / \mathrm{mL}$ puromycin for 3 days. The Flag or Flag-FAM98A-expressing 293T cells were lysed and immunoprecipitated with anti-Flag antibody beads (Wako). The immunoprecipitates were mixed with $40 \mu \mathrm{l}$ elusion buffer (25 mm Tris/HCl, $\mathrm{pH} 7.4,150 \mathrm{~mm} \mathrm{NaCl}$ and 1\% SDS) and 
boiled for 5 minutes and separated from beads using a polystyrene column (Thermo Fisher Scientific, Waltham, MA, USA). $40 \mu \mathrm{l}$ eluted samples were made $80 \mu \mathrm{l}$ by adding $40 \mu \mathrm{l}$ wash buffer. SDS was removed from the sample by passing it through resin SDS (-) column (HiPPR Detergent Removal Column, 0.1ml, 24/pk), Thermo Scientific, Waltham, MA, USA. The eluted proteins were reduced, alkylated and digested using DTT (Wako), iodoacetamide (Wako) and trypsin (Promega, Madison, WI, USA), respectively. The peptides were sequenced using the liquid chromatography-tandem mass spectrometry system (Paradigm MS4, Michrom Bioresources, Sacramento, CA, USA; HTS-PAL, CTC Analytics AG, Zwingen, Swiss; LTQ Orbitrap XL, Thermo Scientific, Waltham, MA, USA). The proteins were identified using the Mascot software package (Matrix Science, London, UK). The experiments were repeated twice to validate the results obtained.

\subsection{Proliferation assay}

The cells were transfected with siRNA using Lipofectamine RNAiMAX and cultured in 96-well plates. Then, the next day of transfection was set as day 0 , and the number of viable cells at the indicated time points were evaluated using the Cell Count Kit-8 (Dojindo, Tokyo, Japan) as described previously [22].

\subsection{Colony formation assay}

Anchorage-independent growth assay was performed as described previously [23]. In brief, HCT116 and HT29 cells $(1 \times 104)$ were mixed with $0.36 \%$ agar in DMEM (10\% FBS) and overlaid onto a $0.72 \%$ agarose layer in 6 -well plates. After two weeks of incubation, colonies in five randomly selected fields were counted. Three independent experiments were performed.

\subsection{Statistical analysis}

All data are presented as the mean \pm Standard Deviation (SD). Statistical analysis for cell proliferation, and colony formation was performed by unpaired t-test using Microsoft Office Excel 2013. $P$ values of $<0.05$ were considered statistically significant. The correlation between PRMT1 and FAM98A expression, PRMT1 and FAM98B expression in clinical samples were determined by Pearson correlation coefficient (R) analysis.

\section{Results}

\subsection{PRMT1 and FAM98A are expressed in colorectal cancer tissues}

To evaluate the role of PRMT1 and FAM98A in colorectal cancer progression, we first examined the expression level of PRMT1 as well as FAM98A in various colorectal cancer cells. 
Immunoblot analysis showed that both PRMT1 and its substrate FAM98A were expressed in all the colorectal cancer cell lines examined (Fig. 1A). In the clinical side of the experiment, we checked the mRNA level of PRMT1 as well as FAM98A in human colorectal tissues (normal $(n=7)$ vs. cancer $(n=18))$ using qRT-PCR analysis. PRMT1 mRNA level was significantly increased in human colorectal cancer specimens as compared to the normal specimens. Also, FAM98A mRNA level was significantly increased in colorectal cancer patients' samples as compared to normal colon samples (Fig. 1B). The data from TCGA database (Colorectal Adenocarcinoma (TCGA, Provisional) [24]) was analyzed to determine the alteration of mRNA and protein expression levels of PRMT1 and FAM98A in CRC samples. Among 633 samples analyzed in this study, $17 \%$ and $18 \%$ were the percentages of genetic alterations in PRMT1 and FAM98A respectively (Fig. 1C). Most of these changes included mRNA and protein upregulation in cancer patients. Together, these data showed the overexpression of both PRMT1 and FAM98A in CRC tissue.

Then, to check the correlation between the expression of these two proteins clinically, we analyzed the expression of both PRMT1 and FAM98A in colorectal specimens ( $\mathrm{n}=76)$ using Pearson correlation analysis. Among these samples 26 were normal controls and 50 were colorectal cancer specimens. Fig. 1D exhibited that PRMT1 expression has a significant positive correlation with FAM98A. The data from TCGA database was analyzed to determine the correlation between PRMT1 and FAM98A at mRNA level in clinical CRC specimens by the cBioPortal platform. A significant relationship between PRMT1 and FAM98A at mRNA level was observed, as revealed on the log2-transformed chart (Fig. 1E). With a threshold of $>0.3$ or $<-0.3$ in either Pearson or Spearman score, PRMT1 was positively correlated with FAM98A (Pearson: 0.137, Spearman: 0.180, N=382). Collectively, these findings clearly indicate the direct association of these proteins with colon cancer incidence and progression, and that PRMT1 and FAM98A proteins are associated with each other in function.

\subsection{FAM98A knockdown reduces PRMT1 expression and Arginine methylation}

So far, we showed the positive correlation between PRMT1 and FAM98A in patients with colorectal cancer. To further confirm this correlation, we next investigated whether FAM98A is required for PRMT1 expression. Western blot analysis revealed the significant decrease in the expression of PRMT1 when FAM98A was depleted in HCT116 and HT29 colorectal cancer cells using FAM98A siRNAs. As a result, arginine methylation was also decreased upon the knockdown of FAM98A as detected by the asymmetric pattern of dimethylated arginines (Fig. 
2A). However, knockdown of PRMT1 did not reduce FAM98A expression and slightly decreased the expression of asymmetric dimethylarginine residues especially in HT29 cells (Fig. 2B).

\subsection{Depletion of FAM98A reduces cancer cell proliferation and colony formation}

To check the role of FAM98A in the malignant characteristics of colon cancer cells, we performed cell proliferation and colony formation assays for siCtrl and siFAM98A treated cells. As shown in Fig. 3A, cell proliferation was significantly reduced after FAM98A knockdown in both HCT116 and HT29 cells. Anchorage independent growth (colony formation) is one of the best in vitro indicators of tumorigenicity. Control and FAM98A siRNA transfected cells were cultured on agar. Two weeks later, cell proliferation on agar was evaluated. As shown in Fig. 3B, both the number and size of the colonies were significantly reduced when FAM98A was depleted. Next, we checked the ability of ectopic expression of PRMT1 to restore the malignant characteristics of FMA98A-depleted cells. We established that HCT116 cells constitutively expressed Flag or Flag PRMT1 by retrovirus infection. The level of exogenously expressed Flag PRMT1 was significantly higher than that of endogenous PRMT1 (Fig. 3C). Interestingly, stable expression of PRMT1 significantly increased both the cell proliferation and colony formation after FAM98A knockdown as compared to the FAM98A-depleted cells (Fig. 3D \&E).

\subsection{FAM98B is expressed in colorectal cancer and required for PRMT1 expression}

In our proteomic analysis for PRMT1 binding partners, we obtained FAM98B also as one of several RNA binding proteins. We observed that FAM98B is expressed differentially in different colon cancer cell lines (Fig. 4A). Next, we checked the mRNA level of FAM98B in human colorectal cancer tissues using qRT-PCR analysis. mRNA level of FAM98B was increased in human colorectal cancer specimens $(n=20)$ as compared to normal colon specimens $(n=10)$ (Fig. 4B). In parallel, a significant positive correlation was observed between FAM98B and PRMT1 by analyzing the expression of both PRMT1 and FAM98B in the clinical samples ( $\mathrm{n}=36)$, among which 13 were normal controls and 23 were colorectal cancer specimens, using Pearson Correlation analysis (Fig. 4C). We confirmed this finding by querying the two genes in the data set found in TCGA database [24]. A significant relationship between PRMT1 and FAM98B at mRNA level was observed, as revealed on the log2-transformed chart (Fig. 4D). With a threshold of $>0.3$ or $<-0.3$ in either Pearson or Spearman score, PRMT1 was positively correlated with FAM98B (Pearson: 0.156, Spearman: 0.202, N=382). To further check this association, we checked the in vitro binding between FAM98B and PRMT1 by transiently expressing both proteins in $293 \mathrm{~T}$ cells. We performed immunoprecipitation (IP)-WB analysis by transfecting $1 \mu \mathrm{g}$ of Flag (control) or Flag FAM98B and $1 \mu \mathrm{g}$ of GFP PRMT1 in 293T cells 
cultured in two $3.5 \mathrm{~cm}$ dishes and IP was performed using beads conjugated with anti-flag antibody as described in the materials and methods. We found a strong binding between these two proteins (Fig. 4E). More interestingly, knockdown of FAM98B reduced the expression of PRMT1 and the methylation pattern in the substrate proteins more efficiently in HCT116 compared to that of HT29 cells (Fig. 4F).

Furthermore, to check the redundancy in function between FAM98A and FAM98B, we overexpressed FAM98A in HCT116 by infecting the cells with Flag FAM98A (Fig. 5A). Then, we checked whether in FAM98A-overexpressing cells, PRMT1 level is reduced by FAM98B depletion. Surprisingly, whereas PRMT1 level is declined in Flag cells, its level has not changed in FAM98A-overexpressing cells upon knockdown of FAM98B (Fig. 5B). The results from TCGA database revealed that there is a significant positive correlation (Pearson: 0.217, Spearman: 0.206, N=382) between FAM98A and FAM98B, as showed on the log2-transformed chart (Fig. 5C). Also, the TCGA analysis revealed significant co-occurrence between PRMT1, FAM98A and FAM98B in the clinical samples of the data set [24] at the mRNA and protein levels (Fig. 5D). Collectively, these findings clearly indicate the direct association of these proteins with colon cancer incidence and progression, and that PRMT1 and FAM98A proteins are associated with each other in function.

\subsection{Knockdown of FAM98B reduces cancer cell proliferation and colony formation}

To further dissect the role of FAM98B in colon cancer cells' progression, we performed cell proliferation and colony formation assays after knockdown of FAM98B. Depletion of FAM98B significantly reduced the cancer cells' proliferation as well as its ability to grow in anchorageindependent conditions (colony formation) (Fig. 6A \& 6B). Importantly, we further checked whether the concurrent depletion of FAM98A and FAM98B has a more diminishing effect on the proliferation and colony formation. As expected, we could not observe further reduction in cell proliferation or colony formation by knocking down both FAM98A and FAM98B (Fig. 6A \& $6 \mathrm{~B})$.

\subsection{FAM98B is a binding partner of FAM98A}

To obtain a more in-depth insight into how the activities of these two proteins are related to each other, we first investigated whether FAM98A binds to FAM98B. We performed IP-WB analysis by transfecting $1 \mu \mathrm{g}$ of Flag (control) or Flag FAM98A and $1 \mu \mathrm{g}$ of GFP FAM98B in $293 \mathrm{~T}$ cells cultured in two $3.5 \mathrm{~cm}$ dishes and IP was performed using beads conjugated with anti-flag antibody as described in the materials and methods. We found that FAM98A and 
FAM98B are bound to each other in vitro (Fig. 7A). In addition, FAM98B not FAM98A was found to form a homodimer as clearly noticed by the co-precipitation of Flag FAM98B and GFP FAM98B after Flag immuno-precipitation (Fig. 7B \& C). FAM98B is a structural homolog of FAM98A, whose glycine-rich $\mathrm{C}$ terminus is much shorter than FAM98A, but their amino acid sequences are very much similar (Fig. 7D). Due to the lack of 3D structures of FAM98A \& B in literature, we used the server SWISS Model to theoretically expect the more likely structure of FAM98A. FAM98A has N-terminus residues spanning around 80 amino acids that through our analysis, the software predicted formation of two helices (Fig. 7E). However, the remaining residues (>80) contain abnormally abundant poly-G and poly-Q that hinder the formation of any stable ternary structure.

These findings led us to check whether FAM98A associates with other binding partners of FAM98B. We performed mass spectrometry analysis of Flag-FAM98A as bait to check its binding partners. Flag (control) or Flag-FAM98A-expressing 293T cells were lysed and immunoprecipitated with anti-Flag antibody beads. The MS experiment was performed twice and compared to the control Flag expressing cells as control. After analyzing the results from mass spectrometry analysis of FAM98A, the binding partners detected included DDX1, C14orf166, HSPC117 and other RNA binding proteins (Supplementary Table 1). The table includes all the binding proteins obtained different from control Flag cells including the score and number of matches. Evidence indicates that, C14orf166 associates with DDX1-HSPC117FAM98B in a novel transcription dependent shuttling RNA-transporting complex. Previous report also showed interaction of C14orf166 with FAM98A and FAM98B [25]. Hence, we checked whether FAM98A also binds with these proteins. We found that FAM98A binds to both DDX1 and C14orf166 but not with HSPC117 (Fig. 8A). To further confirm these results, we prepared deletion mutation constructs of FAM98A and found that both $\mathrm{N}$ terminal (1-329) and C terminal (329-518) FAM98A were required for binding to DDX1 and C14orf166 (Fig. 8B).

\section{Discussion}

In this report, we showed that FAM98A associates with DDX1-C14orf166-FAM98B in a novel complex involved in colorectal cancer progression. Moreover, we have found that PRMT1, FAM98A and FAM98B are highly expressed in colorectal cancer tissues as compared to normal colon tissues. FAM98A was identified as a novel substrate of PRMT1 associated with malignancy in ovarian cancer cell according to our recent report [15]. To examine the role of FAM98A \& B for colorectal cancer progression, we depleted FAM98A \& B expression in HCT116 
and HT29 cells using siRNA knockdown strategy. Consistent with our previous report, we observed a significant reduction in cell proliferation and colony formation in the cancer cells. Although physiological functions of FAM98A are totally unknown, our results suggest an important role of FAM98A in colorectal cancer progression. Although the tumor promoting role of FAM98A \& B is clearly noticed in our study, a previous study has identified DDX1 (one of FAM98A binding partners) as a key modulator protein in miRNA maturation and ovarian tumor suppression [26]. Therefore, depending on the cellular context, expression of DDX1 may promote or suppress the tumor according to partner proteins binding as complex in each type of cancer.

Among proteins identified by our mass spectrometry analysis, FAM98A and its homolog FAM98B were found to be binding partners of PRMT1 [15]. FAM98A and FAM98B are 58\% identical in amino acid sequence and both have multiple RGG/RG motifs. Similar to FAM98A, FAM98B was expressed in all colorectal cancer cells which we examined. The best well-known substrates for PRMT1 are RNA binding proteins involved in various aspects of RNA processing and/or transport, such as hnRNPs, fibrillarin, nucleolin [27,28], and poly(A) binding protein II [29-32]. Most of these substrates contain glycine- and arginine-rich (GAR) sequences that include multiple arginines in RGG or RXR contexts [27, 29, 33]. Arginine methylation mediated by PRMTs affect the functions of the proteins via several mechanisms. There is some evidence that arginine methylation can affect a protein's affinity for RNA [34]. Also, arginine methylation affects the interaction of the substrate proteins with other proteins as well as their subcellular compartmentalization in eukaryotic cells [6].

Recently, it is reported that FAM98B probably as a complex with DDX1, HSPC117 and C14orf166 shuttles between the nucleus and the cytoplasm transporting RNAs suggesting that this complex has a prominent role on nuclear and cytoplasmic RNA fate [25]. The knockdown of FAM98B decreases DDX1 accumulation [33] and the knockdown of C14orf166 decreases DDX1 and FAM98B accumulation [28]. On the other hand, the role of C14orf166 in controlling tumor progression has been previously documented in bladder, breast, uterine cervical cancer, nasopharyngeal carcinoma as well as pelvic lymph node metastasis [35-39].

Here, we showed that at least PRMT1, FAM98A and FAM98B are required to promote the malignancy in colorectal cancer cells. It seems that these proteins work in a complex together as depletion of either FAM98A or FAM98B inhibited the expression of PRMT1 which in turn reduced the asymmetric dimethylarginine (ADMA) in substrate proteins. Silencing of FAM98A decreased PRMT1 expression in HCT116 and HT29, and ADMA signaling in HCT116 cells. In 
this report, we have proved at least in vitro binding among these three proteins. Moreover, we have identified some additional binding partners of these proteins whose tumor controlling role had already been established. It is very likely that the same protein complex can work in different kind of tumors. Consistently, ectopic expression of PRMT1 in FAM98A-depleted cells partially restored the proliferation and colony formation of the cells. Because FAM98A and FAM98B are 58\% identical in amino acid sequence, they can do similar functions. As expected, ectopic expression of FAM98A in HCT116 cells could restore the PRMT1 expression in FAM98B-depleted cells. Similarly, knock down of FAM98A \& B has similar effect on cell proliferation and colony formation when compared with the effect of knocking down FAM98B only suggesting that both proteins work by inhibiting PRMT1 expression.

In summary, as an extension to our previous report, these results would provide further evidence about the role of PRMT1, FAM98A and FAM98B in colorectal cancer cell progression. Further, it would encourage the possibility of studying the molecular interaction of these proteins in vivo after elucidating the $3 \mathrm{D}$ conformational structures by X-ray crystallography and NMR. Collectively, this could shed light on the role of these proteins in colorectal cancer tumorigenesis and on its participation in the sequential phases of RNA metabolism.

\section{References}

1) Siegel, R., Desantis, C., Jemal, A., 2014. Colorectal cancer statistics. CA. Cancer. J. Clin. 64(2), 104-117.

2) Ferlay, J., Soerjomataram, I., Dikshit, R., Eser, S., Mathers, C., Rebelo, M., et al., 2015. Cancer incidence and mortality worldwide: sources, methods and major patterns in GLOBOCAN 2012. Int. J. Cancer. 136(5), E359-386.

3) Zauber, AG., 2015. The impact of screening on colorectal cancer mortality and incidence: has it really made a difference? Dig. Dis. Sci. 60(3), 681-691.

4) Bedford, MT., Clarke, SG., 2009. Protein arginine methylation in mammals: who, what, and why. Mol. Cell. 33(1), 1-13.

5) Debler, EW., Jain, K., Warmack, RA., Feng, Y., Clarke, SG., Blobel, G., et al., 2016. A glutamate/aspartate switch controls product specificity in a protein arginine methyltransferase. Proc. Natl. Acad. Sci. USA. 113(8), 2068-2073. 
6) Yang, Y., Bedford, MT., 2013. Protein arginine methyltransferases and cancer. Nat. Rev. Cancer. 13(1), 37-50.

7) Li, L., Zhang, Z., Ma, T., Huo, R., 2016. PRMT1 regulates tumor growth and metastasis of human melanoma via targeting ALCAM. Mol. Med. Rep. 14(1), 521-528.

8) Seligson, DB., Horvath, S., Shi, T., Yu, H., Tze, S., Grunstein, M., et al., 2005. Global histone modification patterns predict risk of prostate cancer recurrence. Nature. 435(7046), 1262-1266.

9) Cheung, N., Chan, LC., Thompson, A., Cleary, ML., So, CW., 2007. Protein argininemethyltransferase-dependent oncogenesis. Nat. Cell. Biol. 9(10), 1208-1215.

10) Mathioudaki, K., Papadokostopoulou, A., Scorilas, A., Xynopoulos, D., Agnanti, N., Talieri, M., 2008. The PRMT1 gene expression pattern in colon cancer. Br. J. Cancer. 99(12), 2094-2099.

11) Le romancer, M., Treilleux, I., Bouchekioua-bouzaghou, K., Sentis, S., Corbo, L., 2010. Methylation, a key step for nongenomic estrogen signaling in breast tumors. Steroids. 75(8-9), 560-564.

12) Yoshimatsu, M., Toyokawa, G., Hayami, S., Unoki, M., Tsunoda, T., Field, HI., 2011. Dysregulation of PRMT1 and PRMT6, Type I arginine methyltransferases, is involved in various types of human cancers. Int. J. Cancer. 128(3), 562-573.

13) Mathioudaki, K., Scorilas, A., Ardavanis, A., Lymberi, P., Tsiambas, E., Devetzi, M., 2011. Clinical evaluation of PRMT1 gene expression in breast cancer. Tumour Biol. 32(3), $575-582$.

14) Zou, L., Zhang, H., Du, C., Liu, X., Zhu, S., Zhang, W., et al., 2012. Correlation of SRSF1 and PRMT1 expression with clinical status of pediatric acute lymphoblastic leukemia. J. Hematol. Oncol. 5:42.

15) Akter, KA., Mansour, MA., Hyodo, T., Ito, S., Hamaguchi, M., Senga, T., 2016. FAM98A is a novel substrate of PRMT1 required for tumor cell migration, invasion and colony formation. Tumour. Biol. 37(4), 4531-4539. doi: 10.1007/s13277-015-4310-5.

16) Gao, Y., Zhao, Y., Zhang, J., Lu, Y., Liu, X., Geng, P., et al., 2016. The dual function of PRMT1 in modulating epithelial-mesenchymal transition and cellular senescence in breast cancer cells through regulation of ZEB1. Sci. Rep. 6, 19874.

17) Goulet, I., Gauvin, G., Boisvenue, S., Côté, J., 2007. Alternative splicing yields protein arginine methyltransferase 1 isoforms with distinct activity, substrate specificity, and subcellular localization. J. Biol. Chem. 282(45), 33009-33021. 
18) Bedford, MT., Richard, S., 2005. Arginine methylation an emerging regulator of protein function. Mol Cell. 18(3), 263-272.

19) Baldwin, RM., Morettin, A., Côté, J., 2014. Role of PRMTs in cancer: Could minor isoforms be leaving a mark? World. J. Biol. Chem. 5(2), 115-129.

20) Mansour, MA., Hyodo, T., Akter, KA., Kokuryo, T., Uehara, K., Nagino, M., Senga, T., 2016. SATB1 and SATB2 play opposing roles in c-Myc expression and progression of colorectal cancer. Oncotarget. 7(4), 4993-5006.

21) Ayesha, AK., Hyodo, T., Asano, E., Sato, N., Mansour, MA., Ito, S., et al., 2016. UBE2S is associated with malignant characteristics of breast cancer cells. Tumour. Biol. 37(1), 763-772. doi: 10.1007/s13277-015-3863-7.

22) Mansour, MA., Hyodo, T., Ito, S., Kurita, K., Kokuryo, T., Uehara, K., et al., 2015. SATB2 suppresses the progression of colorectal cancer cells via inactivation of MEK5/ERK5 signaling. FEBS. J. 282(8), 1394-1405.

23) Mansour, MA., Asano, E., Hyodo, T., Akter, KA., Takahashi, M., Hamaguchi, M., Senga, T., 2015. Special AT-rich sequence-binding protein 2 suppresses invadopodia formation in HCT116 cells via palladin inhibition. Exp. Cell. Res. 332(1), 78-88.

24) The Cancer Genome Atlas. Colorectal Adenocarcinoma (TCGA, Provisional). http://www.cbioportal.org/study?id=coadread_tcga\#summary.

25) Pérez-gonzález, A., Pazo, A., Navajas, R., Ciordia, S., Rodriguez-frandsen, A., Nieto, A., 2014. hCLE/C14orf166 associates with DDX1-HSPC117-FAM98B in a novel transcription-dependent shuttling RNA-transporting complex. PLoS. ONE. 9(3), e90957.

26) Han, C., Liu, Y., Wan, G., Choi, HJ., Zhao, L., Ivan, C., et al., 2014. The RNA-binding protein DDX1 promotes primary microRNA maturation and inhibits ovarian tumor progression. Cell reports. 8(5), 1447-1460. doi:10.1016/j.celrep.2014.07.058.

27) Gary, JD., Clarke, S., 1998. RNA and protein interactions modulated by protein arginine methylation. Prog. Nucleic. Acid. Res. Mol. Biol. 61, 65-131.

28) Zhang, X., Cheng, X., 2003. Structure of the predominant protein arginine methyltransferase PRMT1 and analysis of its binding to substrate peptides. Structure. 11(5), 509-520. 
29) Smith, JJ., Rücknagel, KP., Schierhorn, A., Tang, J., Nemeth, A., Linder, M., et al., 1999. Unusual sites of arginine methylation in Poly(A)-binding protein II and in vitro methylation by protein arginine methyltransferases PRMT1 and PRMT3. J. Biol. Chem. 274(19), 13229-13234.

30) Stallcup, MR., 2001. Role of protein methylation in chromatin remodeling and transcriptional regulation. Oncogene. 20(24), 3014-3020.

31) Wada, K., Inoue, K., Hagiwara, M., 2002. Identification of methylated proteins by protein arginine $\mathrm{N}$-methyltransferase 1, PRMT1, with a new expression cloning strategy. Biochim Biophys Acta. 1591(1-3), 1-10.

32) Cheng, X., Collins, RE., Zhang, X., 2005. Structural and sequence motifs of protein (histone) methylation enzymes. Annu. Rev. Biophys. Biomol. Struct. 34, 267-294.

33) Popow, J., Englert, M., Weitzer, S., Schleiffer, A., Mierzwa, B., Mechtler, K., et al., 2011. HSPC117 is the essential subunit of a human tRNA splicing ligase complex. Science. 331(6018), 760-764.

34) Rajpurohit, R., Paik, WK., Kim, S., 1994. Effect of enzymic methylation of heterogeneous ribonucleoprotein particle A1 on its nucleic-acid binding and controlled proteolysis. Biochem. J. 304( Pt 3), 903-909.

35) Chen, M., Ye, Y., Zou, B., Guo, S., Zhou, F., Lu, K., et al., 2016. C14orf166 is a highrisk biomarker for bladder cancer and promotes bladder cancer cell proliferation. J. Transl. Med. 14-55. doi:10.1186/s12967-016-0801-4.

36) Cheang, T., Zhou, H., Chen, W., Zhang, B., Liu, L., Yang, J., et al., 2016. C14orf166 overexpression correlates with tumor progression and poor prognosis of breast cancer. J. Transl. Med. 14-54. doi:10.1186/s12967-016-0805-0.

37) Zhang, W., Ou, J., Lei, F., Hou, T., Wu, S., Niu, C., et al., 2016. C14ORF166 overexpression is associated with pelvic lymph node metastasis and poor prognosis in uterine cervical cancer. Tumour. Biol. 37(1), 369-379. doi:10.1007/s13277-015-3806-3.

38) Yang, L., Li, F., Lei, F., Wang, Y., Wu, S., Song, L., Chen, Y., 2015. Overexpression of chromosome 14 open reading frame 166 correlates with disease progression and poorer 
prognosis in human NPC. Tumour. Biol. 36(10), 7977-7986. doi: 10.1007/s13277-0153518-8.

39) Cui, Y., Wu, J., Zong, M., Song, G., Jia, Q., Jiang, J., Han, J., 2009. Proteomic profiling in pancreatic cancer with and without lymph node metastasis. Int. J. Cancer. 1;124(7), 1614-1621. doi: 10.1002/ijc.24163.

\section{Figure Legends}

Figure 1. PRMT1 and FAM98A are expressed in colorectal cancer. (A) The expression of PRMT1 and FAM98A in colorectal cancer cell lines was examined by immunoblot analysis. (B) The level of PRMT1 and FAM98A mRNA in colorectal cancer specimens $(n=18)$ and normal colorectal tissues $(n=7)$ was evaluated by quantitative RT-PCR. The graph indicates the relative PRMT1 and FAM98A mRNA levels. (C) Analysis of the TCGA colorectal adenocarcinoma database (TCGA, Provisional [24]) using cBioPortal showing the percentage and type of PRMT1 \& FAM98A genetic alterations in colorectal cancer. (D) The relative expression levels of PRMT1 and FAM98A mRNA normalized to glyceraldehydes-3-phosphate dehydrogenase (GAPDH) mRNA in 76 clinical tissues (normal controls=26 and CRC specimens $=50$ ) were determined by quantitative RT-PCR. The Pearson correlation coefficient (R) is shown. (E) Analysis of the TCGA colorectal adenocarcinoma database (TCGA, Provisional) using cBioPortal showing the correlation between PRMT1 and FAM98A mRNA levels.

Figure 2. FAM98A is essential for PRMT1 expression and arginine methylation. (A) HCT116 and HT29 cells were transfected with Ctrl and FAM98A siRNAs, and the expression of FAM98A, PRMT1and ADMA was examined $72 \mathrm{~h}$ later by immunoblot analysis. (B) HCT116 and HT29 cells were transfected with Ctrl and PRMT1 siRNAs, and the expression of PRMT1, FAM98A and ADMA was examined $72 \mathrm{~h}$ later by immunoblot analysis.

Figure 3. Knockdown of FAM98A reduces cancer cell proliferation and colony formation in vitro. (A) HCT116 and HT29 cells were transfected with Ctrl and FAM98A siRNAs, and the number of viable cells at the indicated time points was determined using a Cell Counting Kit-8 assay. 
(B) siCtrl or siFAM98A transfected HCT116 and HT29 cells were subjected to a colony formation assay. Representative images are shown, and the graphs indicate the average number and size of colonies per field $\left({ }^{*} \mathrm{P}<0.05\right)$. (C) HCT116 cells constitutively expressing FlagPRMT1 was established by retrovirus infection. The expression of the indicated proteins in the cell line was examined by immunoblotting. The arrow and the arrowhead indicate the endogenous protein and the exogenous protein, respectively. (D) The numbers of viable cells at the indicated time points were determined using a Cell counting Kit-8 assay for HCT116 cells (*P<0.05 ). (E) siCtrl, siFAM98A and siFAM98A/Flag PRMT1 HCT116 cells were subjected to colony formation assay. The graphs indicate the average number of colonies per field $(* \mathrm{P}<0.05)$.

Figure 4. Knockdown of FAM98B reduces PRMT1 expression. (A) The expression of FAM98B in colorectal cancer cell lines was examined by immunoblot analysis. (B) The level of FAM98B mRNA in colorectal cancer specimens $(n=20)$ and normal colorectal tissues $(n=10)$ was evaluated by quantitative RT-PCR. The graph indicates the relative FAM98B mRNA levels. (C) The relative expression levels of PRMT1 and FAM98B mRNA normalized to glyceraldehydes-3phosphate dehydrogenase (GAPDH) mRNA in 36 clinical tissues (normal controls=13 and CRC specimens=23) were determined by quantitative RT-PCR. The Pearson correlation coefficient (R) is shown. (D) Analysis of the TCGA colorectal adenocarcinoma database (TCGA, Provisional) using cBioPortal showing the correlation between PRMT1 and FAM98B mRNA levels. (E) 293T cells cultured in $35 \mathrm{~mm}$ dishes were transfected with $1 \mu \mathrm{g}$ of plasmid encoding Flag (control) or Flag-FAM98B and GFP-PRMT1, and cells were lysed $24 \mathrm{~h}$ later. Flag and Flag-FAM98A cells were immunoprecipitated with beads conjugated with anti-Flag antibody and immunoblotted for anti-Flag and anti-GFP antibodies. (F) HCT116 and HT 29 cells were transfected with Ctrl and FAM98B siRNAs, and the expression of FAM98B, FAM98A, PRMT1 and ADMA was examined $72 \mathrm{~h}$ later.

Figure 5. Ectopic expression of FAM98A restores PRMT1 in FAM98B depleted cells. (A) HCT116 cells constitutively expressing Flag or Flag-FAM98A were established by retrovirus infection and the expression of Flag and FAM98A was checked by immunoblotting. (B) HCT116 cells constitutively expressing Flag or Flag-FAM98A was established by retrovirus infection and transfected with Ctrl and FAM98B siRNAs. Then, the expression of FAM98B and PRMT1 was examined $72 \mathrm{~h}$ later by immunoblotting. (C) Analysis of the TCGA colorectal adenocarcinoma 
database (TCGA, Provisional) using cBioPortal showing the correlation between FAM98A and FAM98B mRNA levels. (D) Analysis of the TCGA colorectal adenocarcinoma database (TCGA, Provisional) using cBioPortal showing the tendency towards co-occurrence between PRMT1, FAM98A and FAM98B.

Figure 6. FAM98A and FAM98B work in a similar axis. (A) HCT116 and HT29 cells were transfected with Ctrl, FAM98B and FAM98A+FAM98B siRNAs, and the number of viable cells at the indicated time points was determined using a Cell Counting Kit-8 assay. (B) siCtrl, siFAM98B or siFAM98A+siFAM98B transfected in HCT116 and HT29 cells were subjected to a colony formation assay. Representative images are shown, and the graphs indicate the average number and size of colonies per field $\left({ }^{*} \mathrm{P}<0.05\right)$.

Figure 7. FAM98A associates with FAM98B. (A) 293T cells cultured in $35 \mathrm{~mm}$ dishes were transfected with $1 \mu \mathrm{g}$ of plasmid encoding Flag (control) or Flag-FAM98A and GFP-FAM98B, and cells were lysed $24 \mathrm{~h}$ later. Flag or Flag-FAM98A was immunoprecipitated with beads conjugated with anti-Flag antibody and immunoblotted for anti-Flag and anti-GFP antibodies. The arrow and arrow-head indicate FAM98A and band of unknown origin related to beads respectively (B) 293T cells cultured in $35 \mathrm{~mm}$ dishes were transfected with $1 \mu \mathrm{g}$ of plasmid encoding Flag (control) or Flag-FAM98A and GFP-FAM98A and cells were lysed $24 \mathrm{~h}$ later. Flag or Flag-FAM98A was immunoprecipitated with beads conjugated with anti-Flag antibody and immunoblotted for anti-Flag and anti-GFP antibodies. The arrow and arrow-head indicate FAM98A and band of unknown origin related to beads respectively. (C) 293T cells cultured in 35 mm dishes were transfected with $1 \mu \mathrm{g}$ of plasmid encoding Flag (control) or Flag-FAM98B and GFP-FAM98B and cells were lysed $24 \mathrm{~h}$ later. Flag or Flag-FAM98B was immunoprecipitated with beads conjugated with anti-Flag antibody and immunoblotted for anti-Flag and anti-GFP antibodies. (D) The glycine-rich C terminus in FAM98A and FAM98B is depicted for both structures. (E) The two helices structures of FAM98A in the first 80 residues of the protein as expected by the server SWISS model.

Figure 8. FAM98A associates with FAM98B binding partners. (A) 293T cells cultured in $35 \mathrm{~mm}$ dishes were transfected with $1 \mu \mathrm{g}$ of plasmid encoding Flag (control) or Flag-FAM98A and GFP- 
DDX1 or GFP-C14orf166, and cells were lysed $24 \mathrm{~h}$ later. Flag or Flag-FAM98A was immunoprecipitated with anti-Flag antibody and immunoblotted for anti-Flag and anti-GFP antibodies. (B) $293 \mathrm{~T}$ cells cultured in $35 \mathrm{~mm}$ dishes were transfected with $1 \mu \mathrm{g}$ of plasmid encoding Flag-DDX1 or Flag-C14orf166 together with GFP, GFP-tagged 1-329 FAM98A or GFPtagged 329-518 FAM98A. Twenty four hours later, cells were lysed and, Flag-DDX1 and Flag C14orf166 were immunoprecipitated with anti-Flag antibody and immunoblotted with anti-Flag and anti-GFP antibodies. 

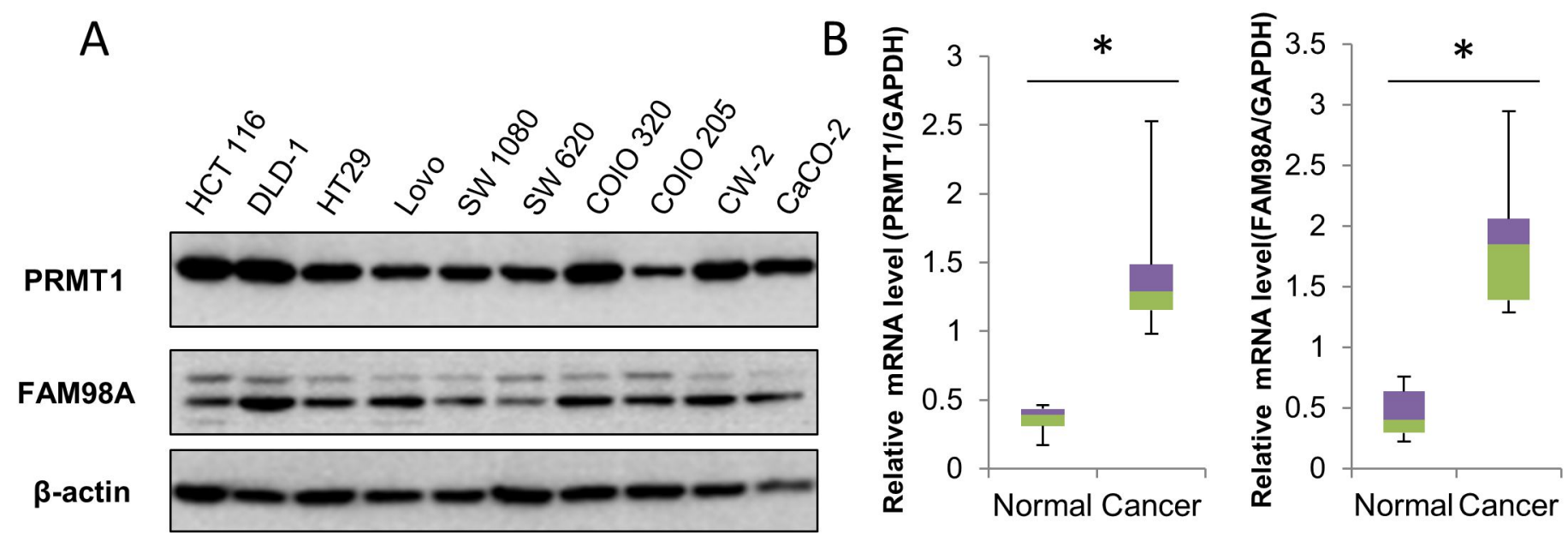

C

\section{Colorectal Adenocarcinoma (TCGA) (633 samples)}

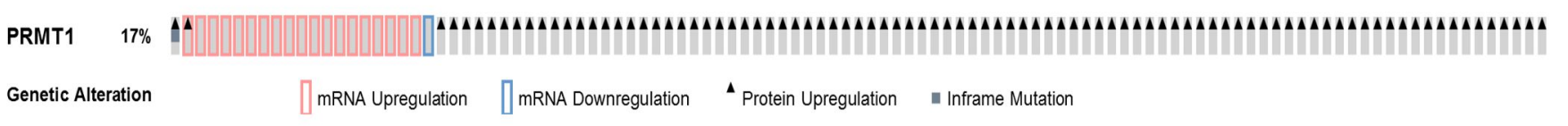

FAM98A $\quad 18 \%$

Genetic Alteration

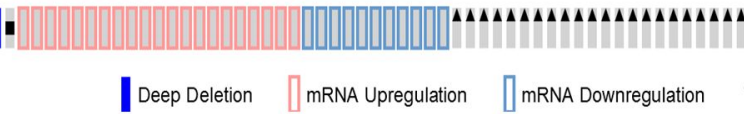

Protein Upregulation

- Truncating Mutation

D

Pearson correlation, $\mathrm{R}=0.74$

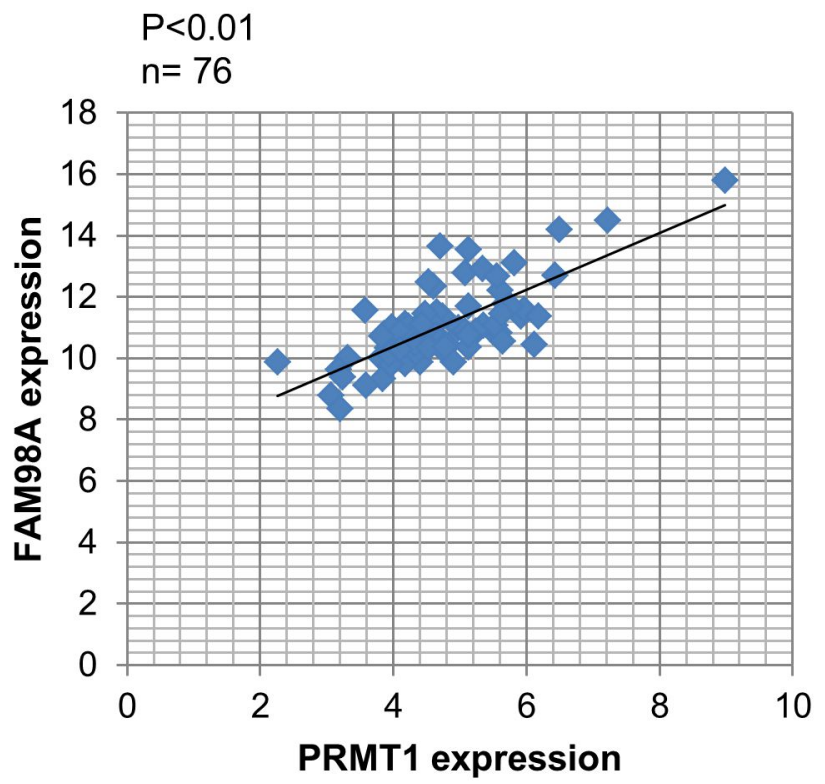

$E$

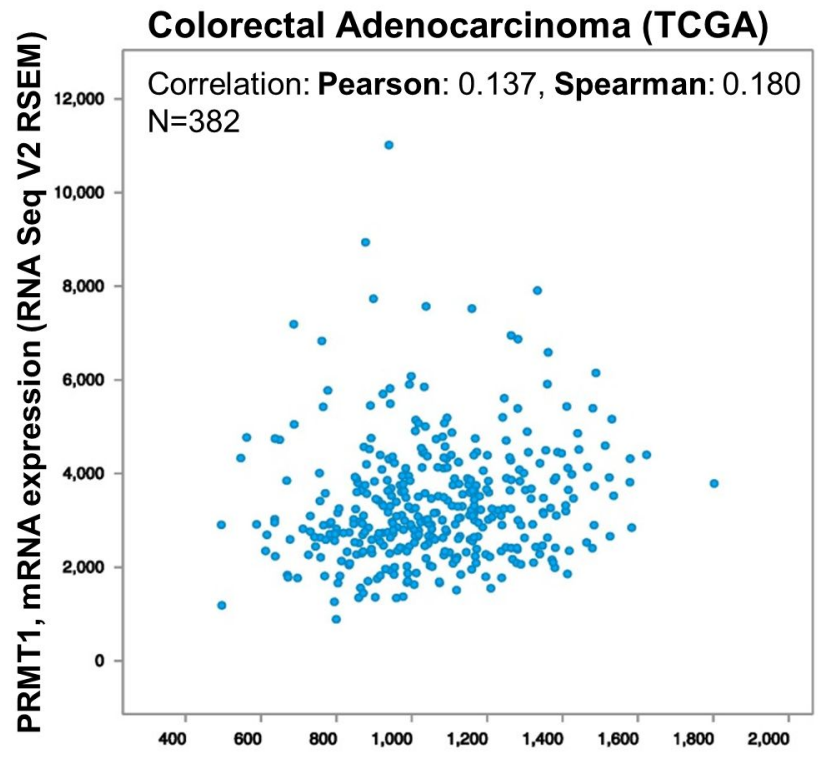

FAM98A, mRNA expression (RNA Seq V2 RSEM) 


\section{Figure 2}

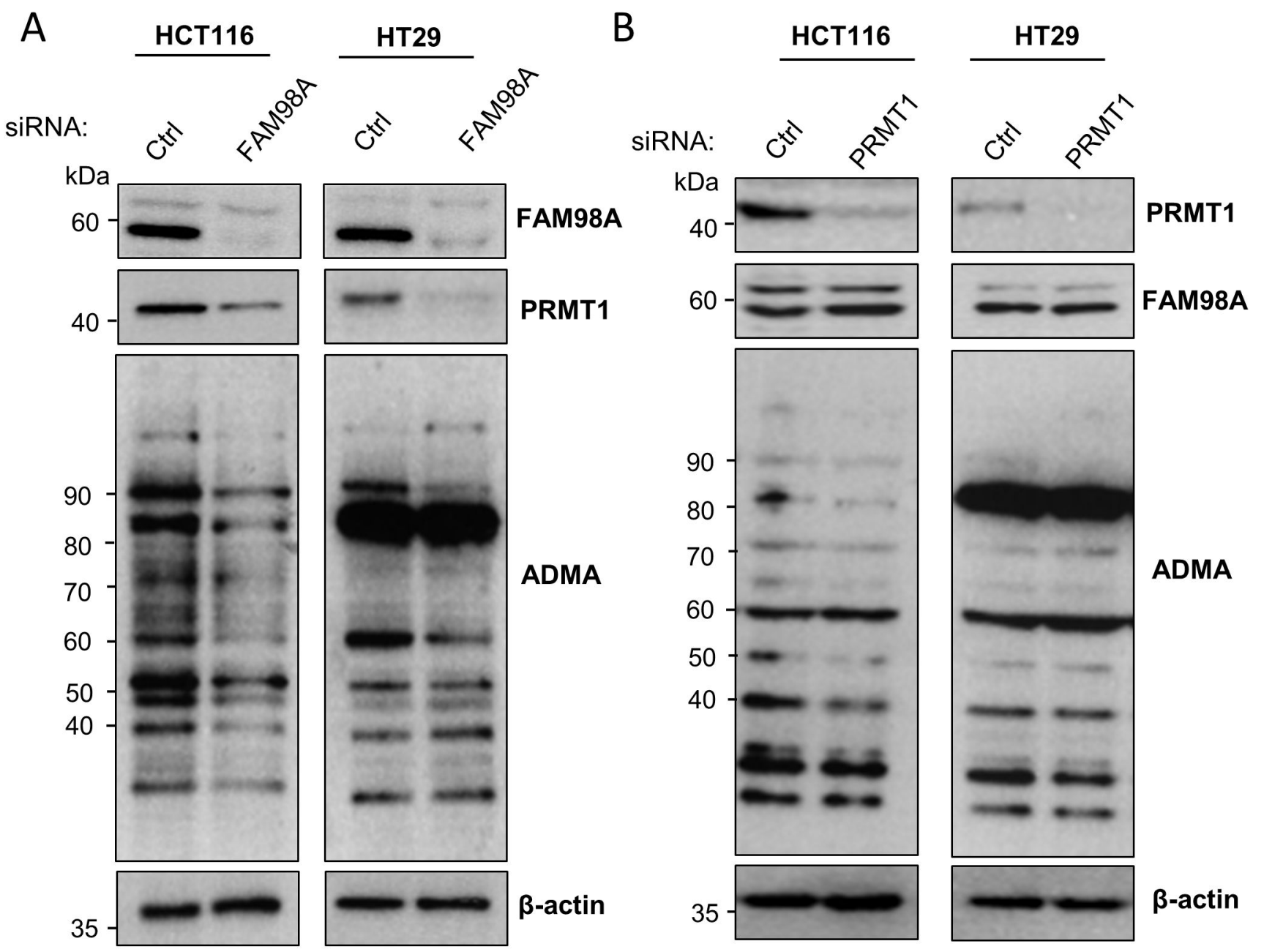


Figure 3
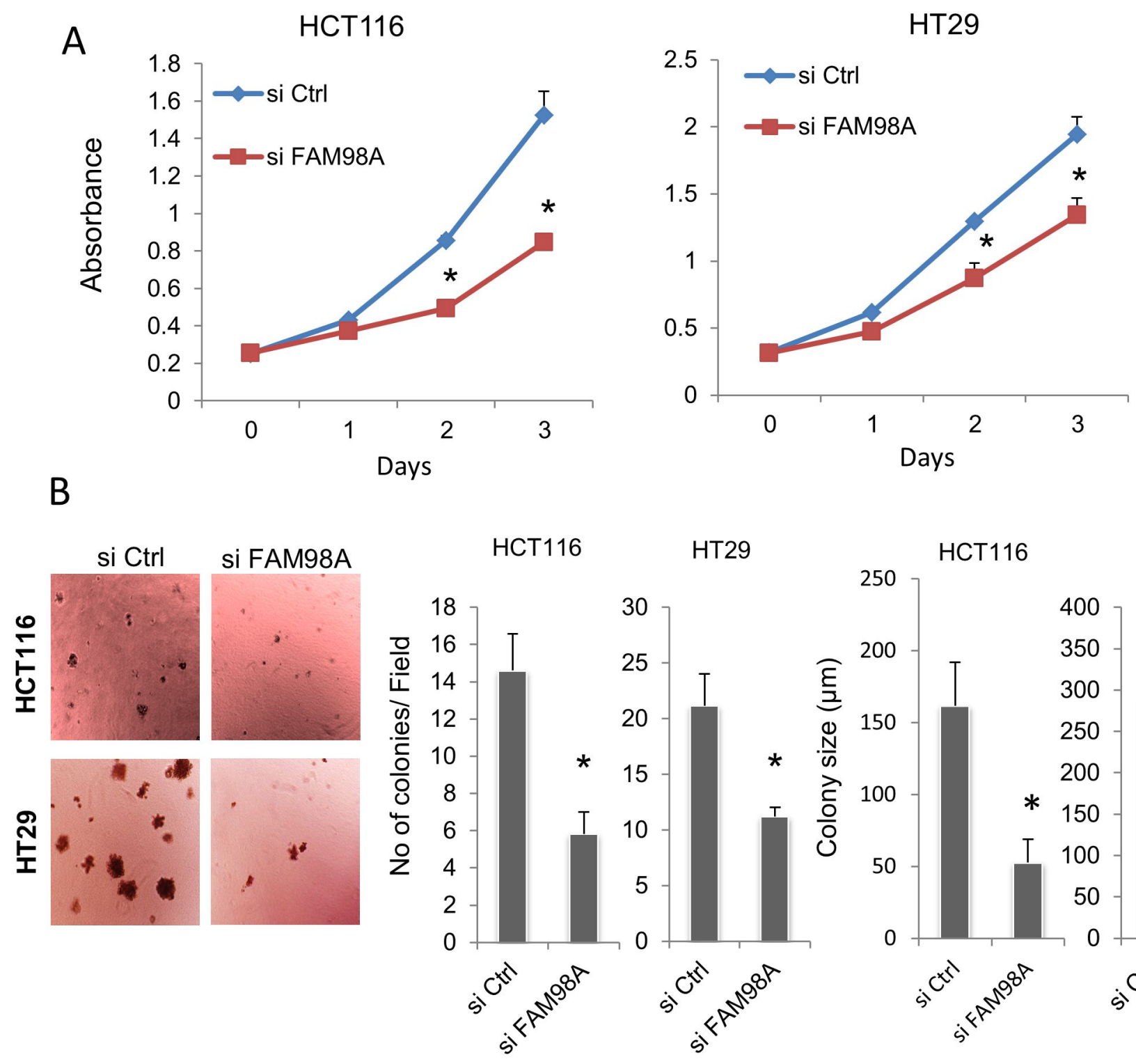

HT29
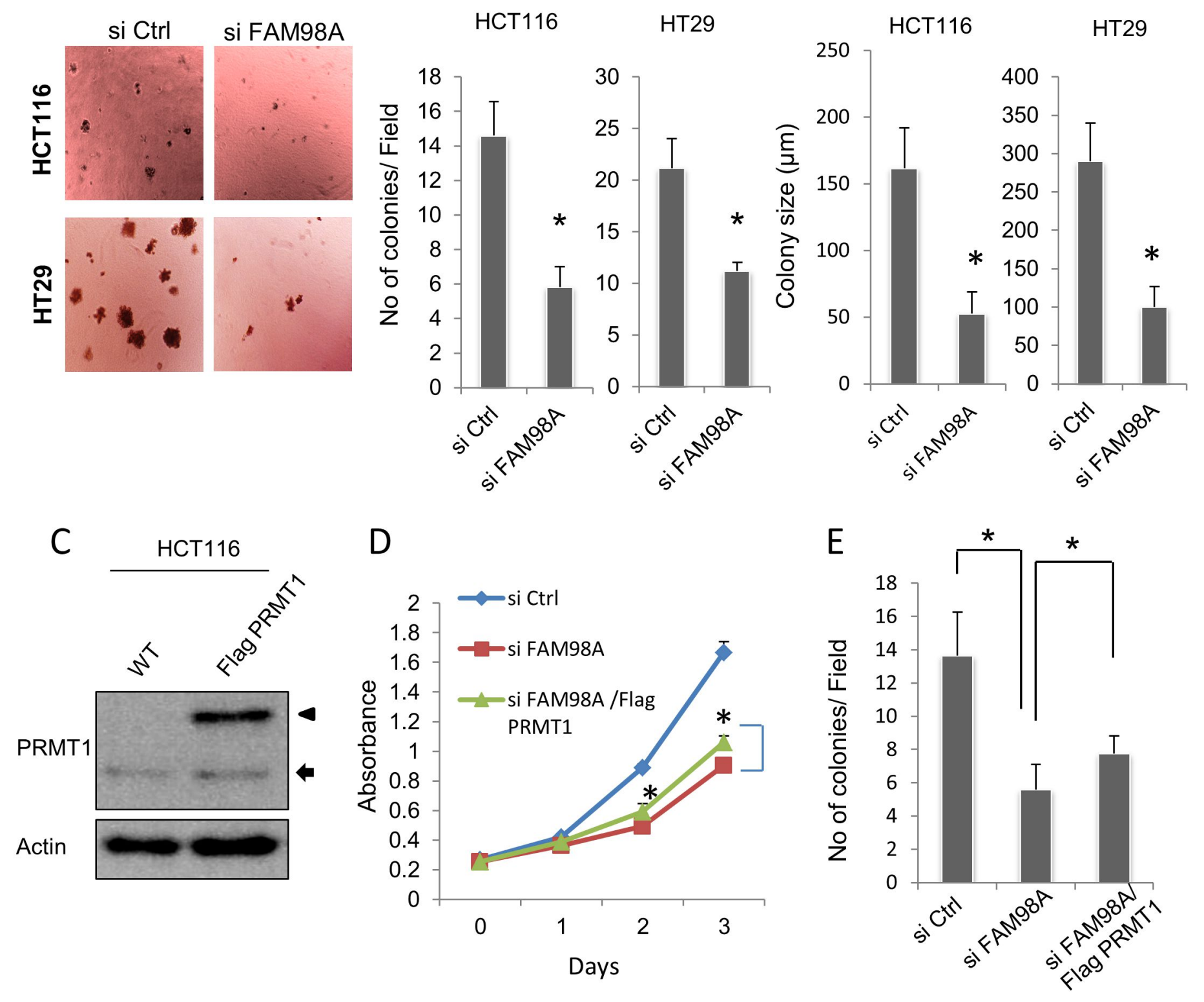
Figure 4

A

FAM98B

Actin

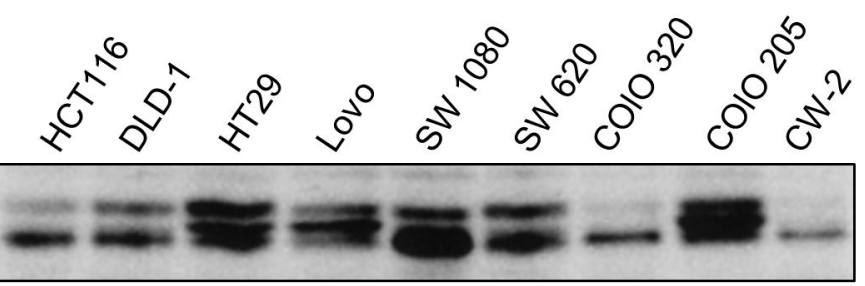

C

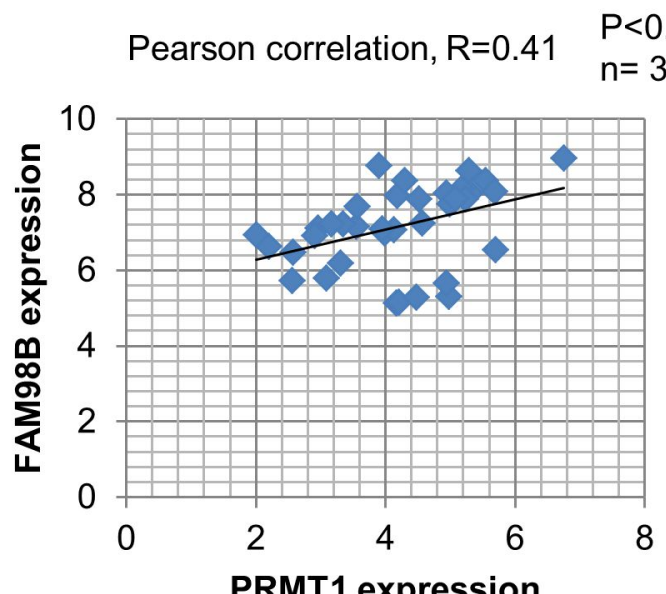

PRMT1 expression

$E$

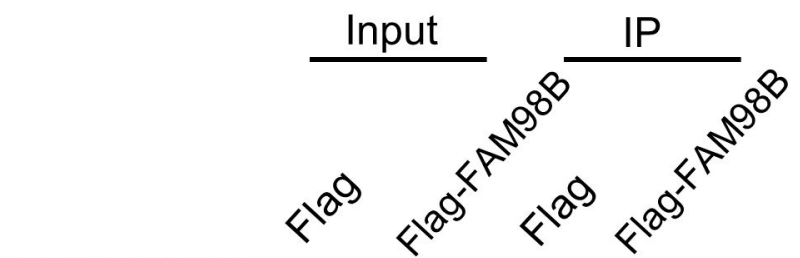

GFP PRMT1: +++

Flag

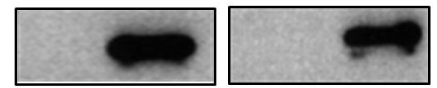

GFP

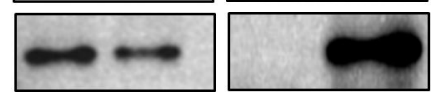

F
B
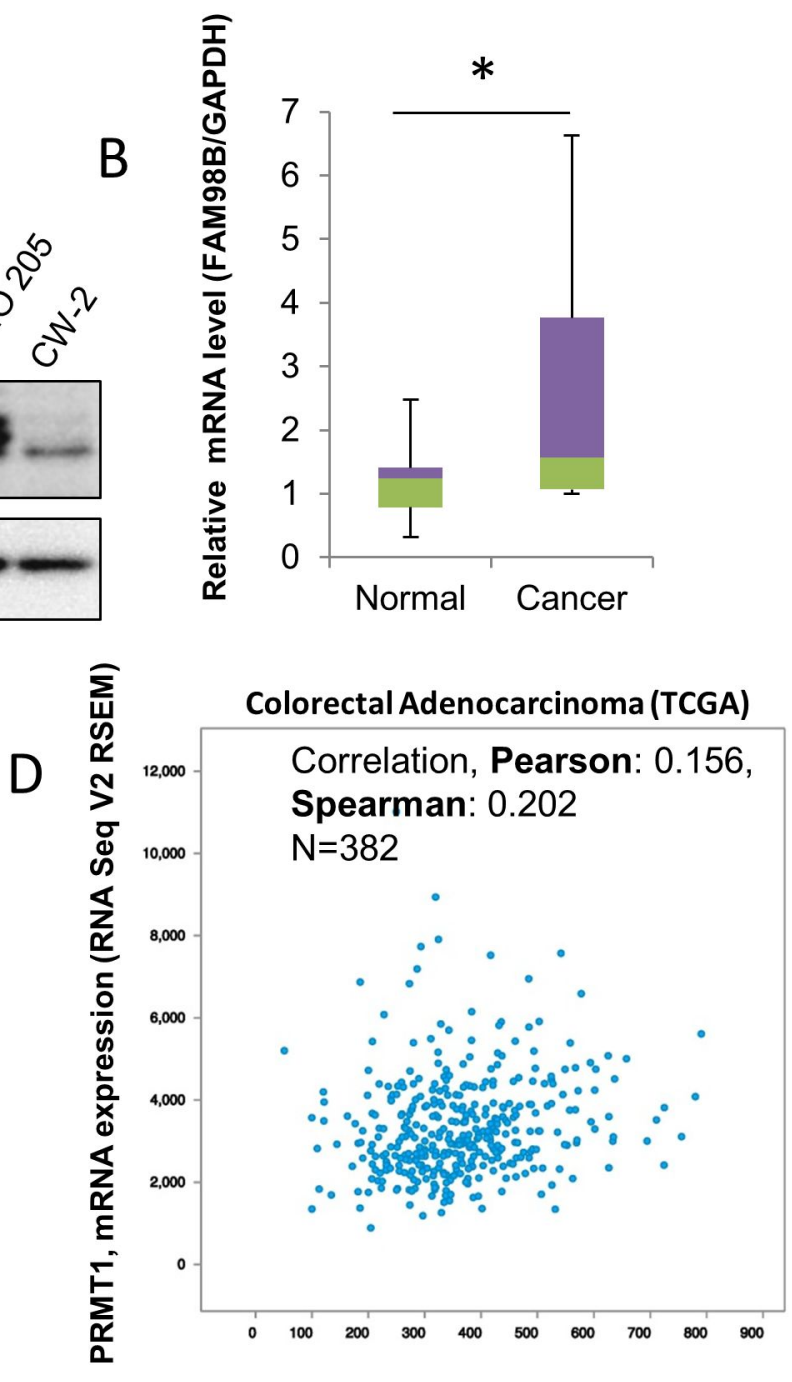

FAM98B, mRNA expression (RNA Seq V2 RSEM)

siRNA:
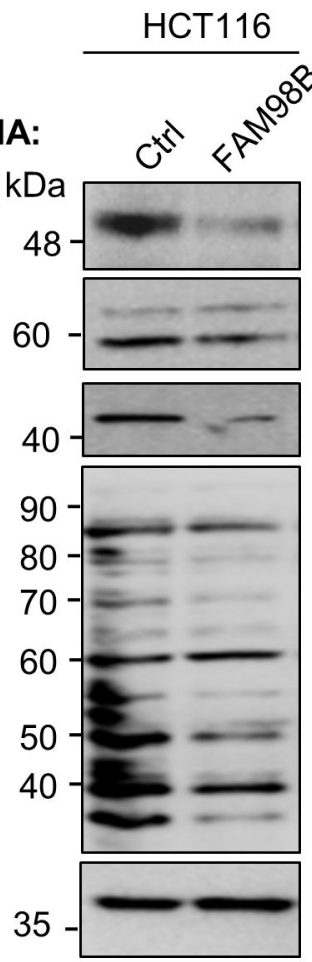

HT29

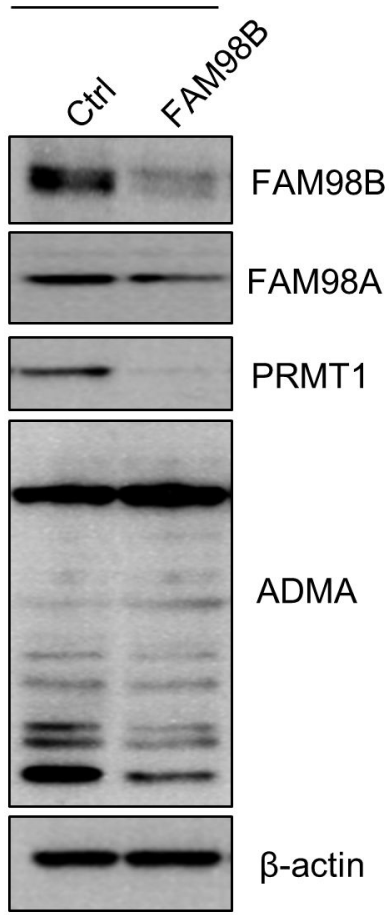


Figure 5

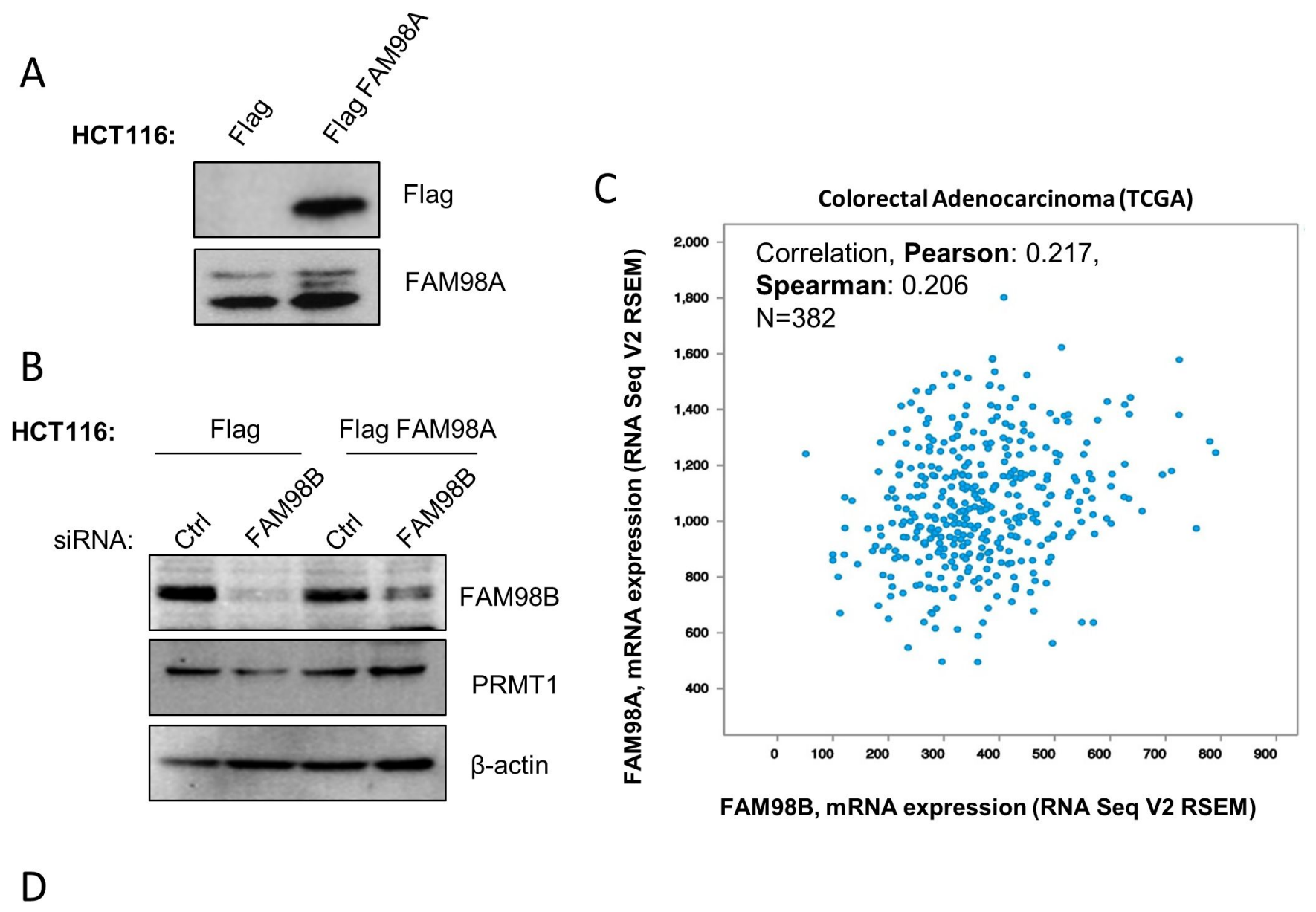

\begin{tabular}{|l|l|l|l|l|}
\hline Gene A & Gene B & P-Value & $\begin{array}{l}\text { Log Odds } \\
\text { Ratio }\end{array}$ & Association \\
\hline PRMT1 & FAM98A & $<0.001$ & $>3$ & $\begin{array}{l}\text { Tendency towards co- } \\
\text { occurrence (Significant) }\end{array}$ \\
\hline PRMT1 & FAM98B & $<0.001$ & $>3$ & $\begin{array}{l}\text { Tendency towards co- } \\
\text { occurrence (Significant) }\end{array}$ \\
\hline FAM98A & FAM98B & $<0.001$ & $>3$ & $\begin{array}{l}\text { Tendency towards co- } \\
\text { occurrence (Significant) }\end{array}$ \\
\hline
\end{tabular}




\section{Figure 6}

A

HCT 116

HT 29
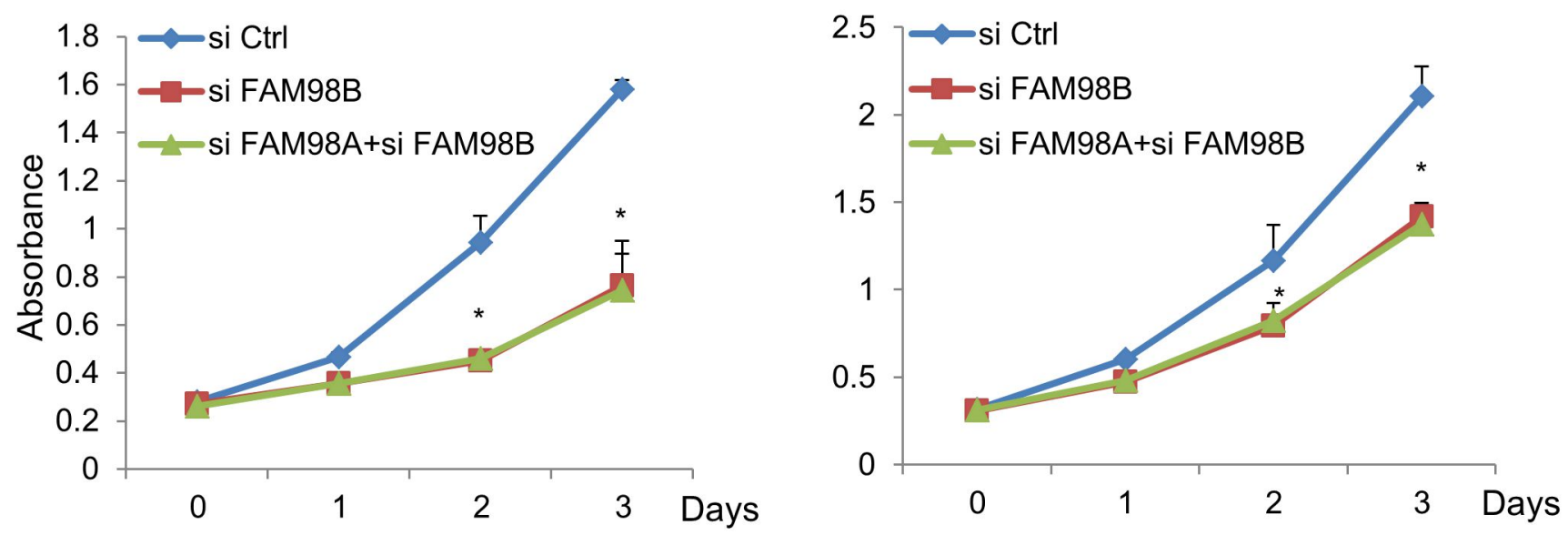

B
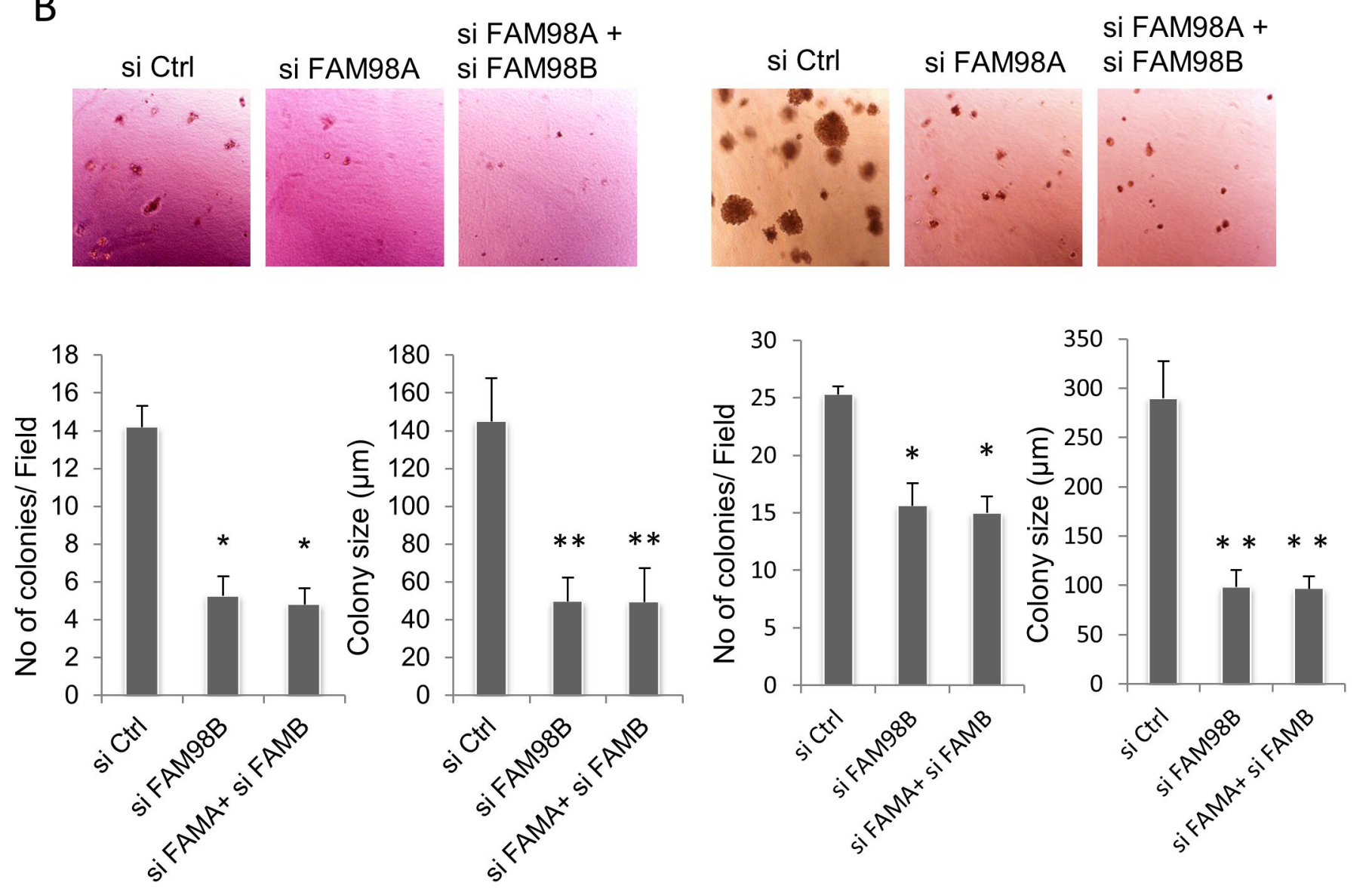


\section{Figure 7}
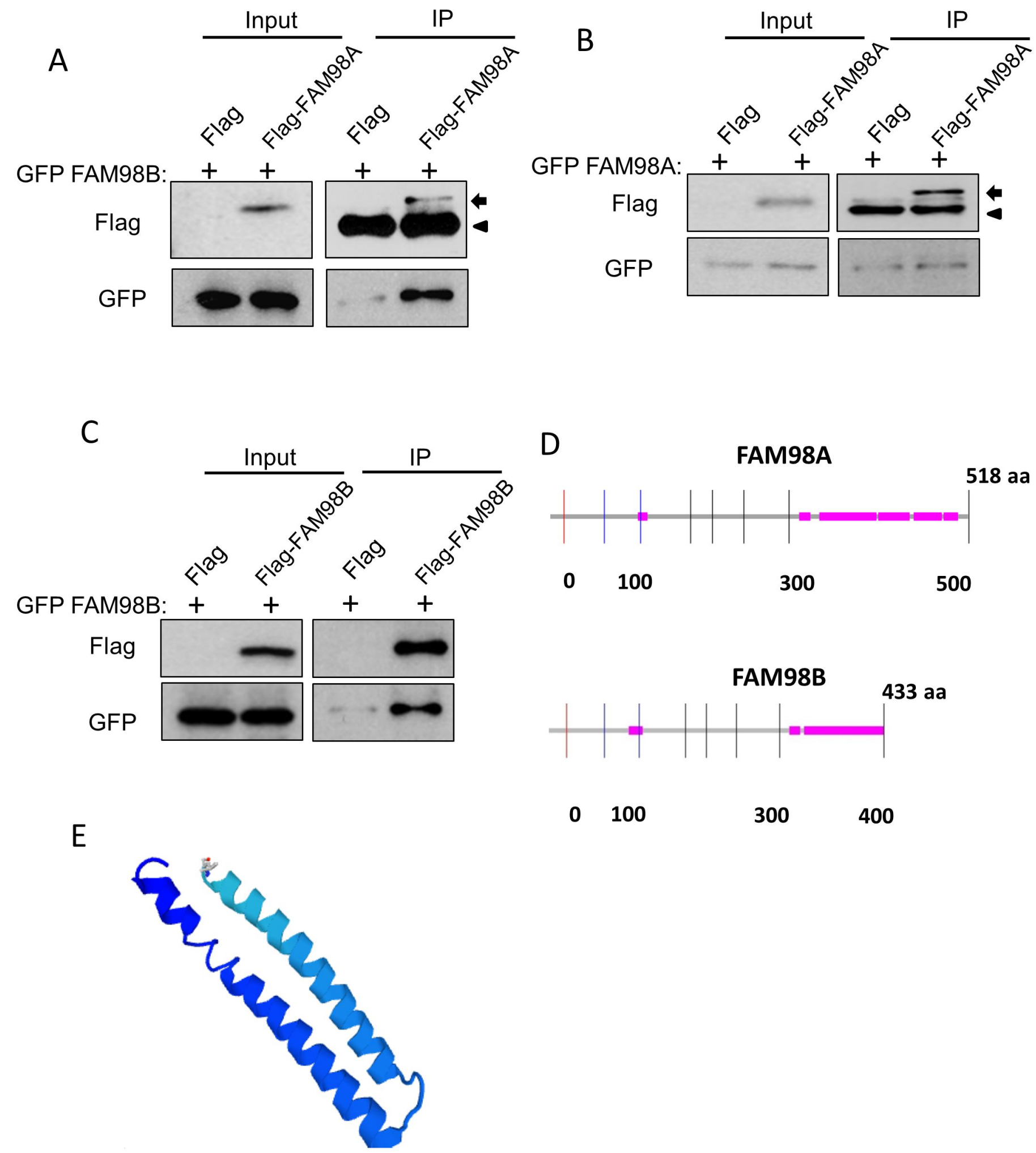

N-terminal (80N)-residue FAM98A 
Figure 8

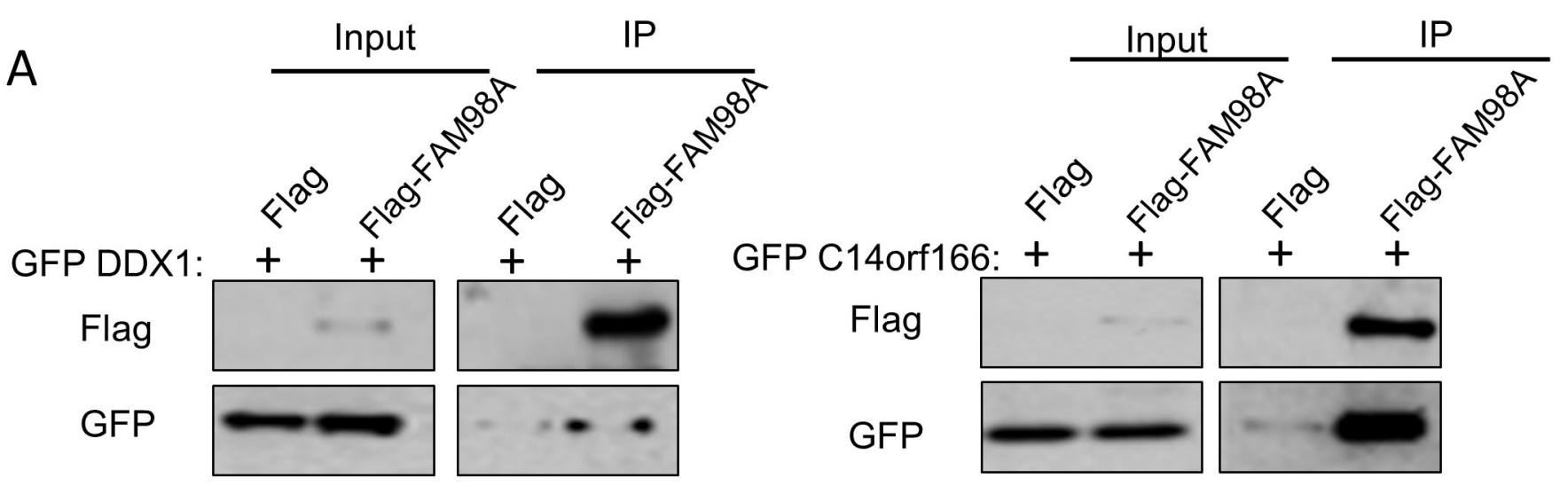

B
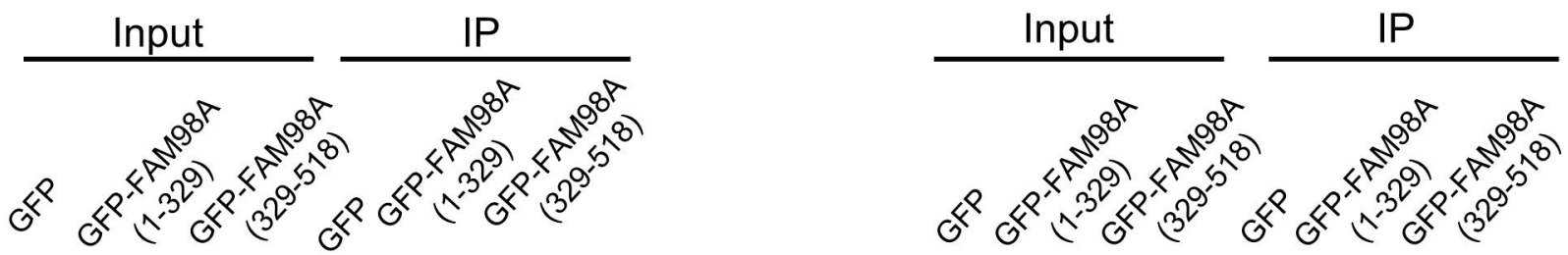

Flag

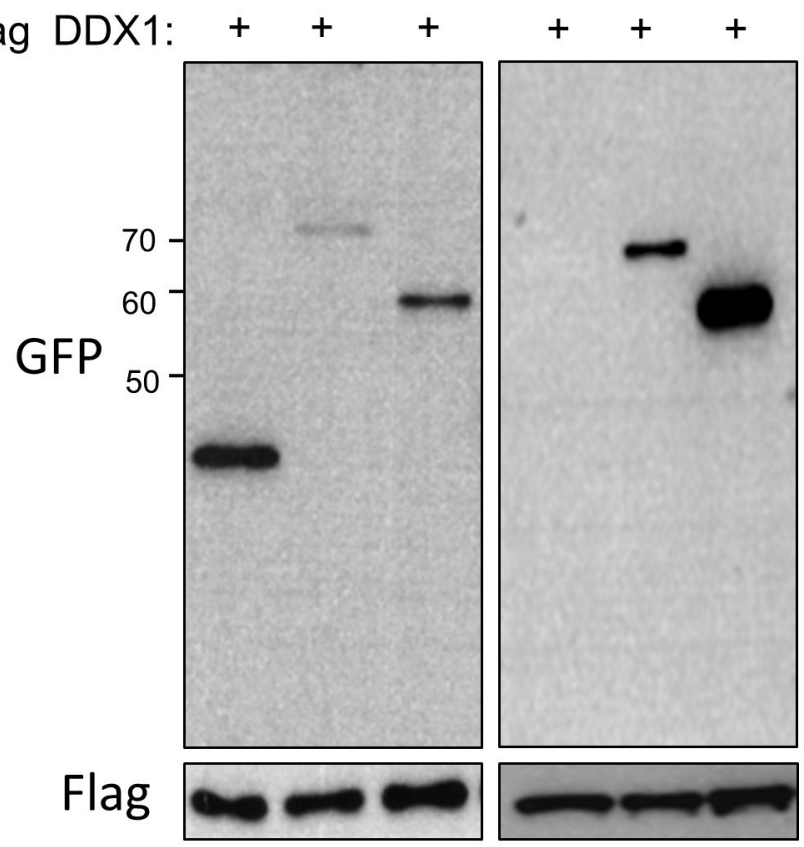

Flag C14orf166: +++

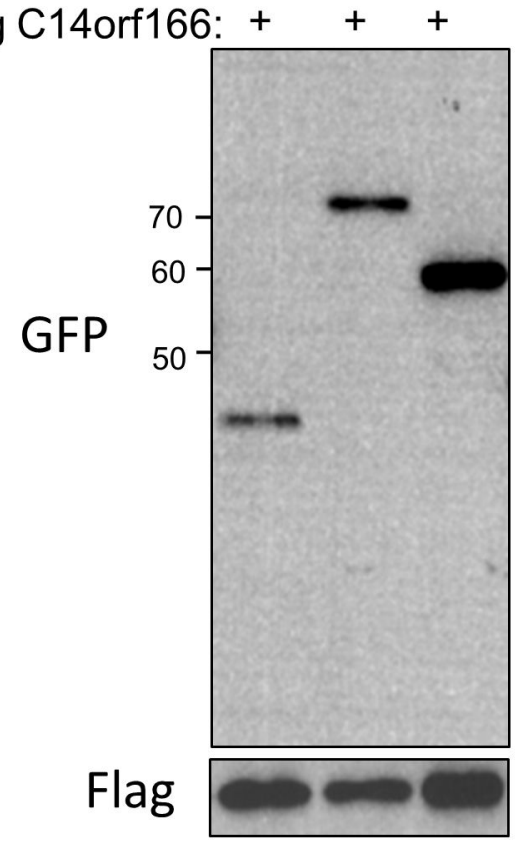

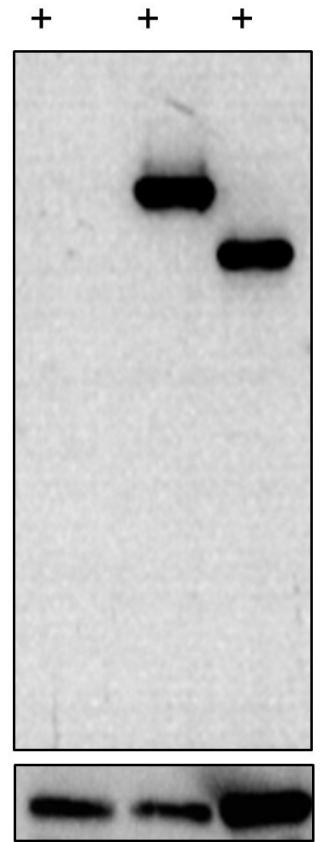


Supplementary Table 1: Binding partners of Flag-FAM98A (bait) in 293T cells by mass spectrometry analysis. The experiment was repeated twice to ensure the validity of the results.

\begin{tabular}{|c|c|c|c|}
\hline Accession & Score & Mass & Num. of matches \\
\hline RTCB_HUMAN & 2272 & 55688 & 158 \\
\hline DDX1_HUMAN & 2038 & 83349 & 185 \\
\hline FA98A_HUMAN & 919 & 55823 & 68 \\
\hline PABP1_BOVIN & 796 & 70854 & 65 \\
\hline PABP4_HUMAN & 438 & 71080 & 26 \\
\hline ATX2L_HUMAN & 626 & 113589 & 77 \\
\hline LG3BP_HUMAN & 318 & 66202 & 29 \\
\hline TBA_XENLA & 277 & 50532 & 12 \\
\hline ATX2_HUMAN & 249 & 140823 & 29 \\
\hline NUFP2_HUMAN & 232 & 76132 & 24 \\
\hline RL18_BOVIN & 225 & 21636 & 9 \\
\hline HSP7C_BOVIN & 225 & 71424 & 13 \\
\hline RL8_BOVIN & 217 & 28235 & 20 \\
\hline ALBU_MOUSE & 155 & 70700 & 11 \\
\hline C1QA_MOUSE & 150 & 26186 & 13 \\
\hline KV3A7_MOUSE & 140 & 12445 & 8 \\
\hline C1QC_MOUSE & 136 & 26374 & 9 \\
\hline RL14_HUMAN & 127 & 23531 & 5 \\
\hline C1QB_MOUSE & 127 & 26929 & 7 \\
\hline CN166_HUMAN & 126 & 28165 & 15 \\
\hline RL7_HUMAN & 123 & 29264 & 13 \\
\hline TBB5_BOVIN & 117 & 50095 & 7 \\
\hline TBB3_CHICK & 106 & 50285 & 9 \\
\hline TBB5_CHICK & 73 & 50395 & 8 \\
\hline RS18_BOVIN & 113 & 17708 & 11 \\
\hline RL10_HUMAN & 107 & 25044 & 6 \\
\hline RL27A_BOVIN & 101 & 16681 & 6 \\
\hline VIME_CHLAE & 98 & 53733 & 13 \\
\hline VIM1_XENLA & 49 & 52869 & 10 \\
\hline LSM12_BOVIN & 97 & 21972 & 8 \\
\hline RL3_HUMAN & 85 & 46365 & 12 \\
\hline RS2_BOVIN & 82 & 31501 & 17 \\
\hline N4BP2_HUMAN & 82 & 200444 & 5 \\
\hline RL17_FELCA & 82 & 21611 & 11 \\
\hline GRP78_BOVIN & 80 & 72470 & 9 \\
\hline RL18A_BOVIN & 77 & 21034 & 4 \\
\hline IGHG3_MOUSE & 77 & 44472 & 10 \\
\hline RL7A_HUMAN & 72 & 30148 & 9 \\
\hline RL19_BOVIN & 72 & 23565 & 4 \\
\hline RL4_BOVIN & 69 & 47667 & 15 \\
\hline DDX-̄3L_MOUSE & 68 & 73494 & 3 \\
\hline RS11_BOVIN & 68 & 18590 & 12 \\
\hline 1433Z_BOVIN & 67 & 27899 & 2 \\
\hline RS6_BOVIN & 64 & 28820 & 10 \\
\hline RS4X_CHLAE & 62 & 29807 & 2 \\
\hline RL27_BOVIN & 59 & 15788 & 6 \\
\hline RL23_BOVIN & 59 & 14970 & 8 \\
\hline RL30_BOVIN & 52 & 12947 & 4 \\
\hline
\end{tabular}




\begin{tabular}{|c|c|c|c|}
\hline CAPR1_BOVIN & 52 & 78451 & 2 \\
\hline RS16_BOVIN & 51 & 16549 & 5 \\
\hline GLYA_LACAC & 51 & 45276 & 4 \\
\hline RL15_BOVIN & 51 & 24245 & 6 \\
\hline RS9_BOVIN & 46 & 22635 & 7 \\
\hline RL13_HUMAN & 45 & 24304 & 4 \\
\hline RS14A_ANOGA & 42 & 16360 & 4 \\
\hline PS4_PINST & 41 & 870 & 6 \\
\hline VP5_BTV1A & 40 & 59378 & 4 \\
\hline TFG_HUMAN & 38 & 43478 & 4 \\
\hline TNR6B_HUMAN & 37 & 194739 & 7 \\
\hline AROK_STAS1 & 37 & 19589 & 18 \\
\hline RL28_BOVIN & 36 & 15740 & 6 \\
\hline RL13A_BOVIN & 36 & 23565 & 3 \\
\hline AXP83_CIOIN & 36 & 84269 & 4 \\
\hline HNRH1_HUMAN & 35 & 49484 & 2 \\
\hline FXR1_HUMAN & 35 & 70020 & 7 \\
\hline KV2A7_MOUSE & 35 & 12379 & 4 \\
\hline ANT1_ASHGO & 34 & 37204 & 2 \\
\hline GCAA_MOUSE & 34 & 36936 & 8 \\
\hline ALBU_HUMAN & 32 & 71317 & 3 \\
\hline Y4QE_RHISN & 32 & 44087 & 5 \\
\hline RS27A_DROME & 32 & 18157 & 3 \\
\hline RS3_BOVIN & 31 & 26842 & 1 \\
\hline RL6_CHILA & 30 & 32652 & 5 \\
\hline DSG1_HUMAN & 29 & 114702 & 5 \\
\hline NMT_CANAL & 29 & 52129 & 5 \\
\hline IGH1M_MOUSE & 28 & 44043 & 2 \\
\hline MATK_ADELA & 28 & 61376 & 7 \\
\hline VIT1_FUNHE & 28 & 189402 & 3 \\
\hline NRDR_ARTAT & 27 & 18528 & 8 \\
\hline PRM1_CANAL & 27 & 70309 & 4 \\
\hline SYC_STAMF & 27 & 56389 & 4 \\
\hline HNRPK_BOVIN & 27 & 51272 & 1 \\
\hline RS23_BOVIN & 25 & 15969 & 8 \\
\hline MTNB_DROGR & 24 & 26587 & 2 \\
\hline KPYK_CANAL & 24 & 55752 & 1 \\
\hline PNRC2_XENTR & 23 & 15266 & 1 \\
\hline KHDR1_HUMAN & 23 & 48311 & 6 \\
\hline RPOA2_METMA & 23 & 45417 & 4 \\
\hline HV304_HUMAN & 22 & 12462 & 1 \\
\hline ENV_HV1V9 & 15 & 98770 & 3 \\
\hline
\end{tabular}

